\title{
Application of the Electro-Optical Kerr Effect in Physical-Chemical Analysis of Binary Systems
}

\author{
O.V. Prezhdo ${ }^{a}$, S.A. Tyurin ${ }^{b}$, M. PisarczyK $^{c}$, A. OdziemeK ${ }^{c}$ \\ AND V.V. PREZHDO ${ }^{c}$ \\ ${ }^{a}$ Department of Chemistry, University of Washington, Seattle, WA 98115, U.S.A. \\ ${ }^{b}$ Department of Physic, Kharkov Fire-Technical Academy \\ 61000 Kharkov, Ukraine \\ ${ }^{c}$ Institute of Chemistry, Jan Kochanowski University \\ Chęcińska 5, 25-020 Kielce, Poland
}

(Received October 25, 2001; in final form February 1, 2002)

\begin{abstract}
Applications of the electro-optical Kerr effect in physical-chemical analysis of binary mixtures are reviewed. Improvements in the experimental techniques, in particular, development of the pulsed and alternating electric field based approaches, have led to significant advances in this area. However, problems associated with the description of the internal field of liquids remain and hamper broader applications of the techniques. It is shown that a further progress in the electro-optical Kerr effect characterization of chemical systems relies on the development of a more general theory of optical properties of condensed phase chemical mixtures that can account for nonlinear effects, such as hyperpolarizability, dispersion and specific conditions of the Kerr constant measurements.
\end{abstract}

PACS numbers: 31.70.Dk, 78.20.Fm, 72.80.Jc

\section{Introduction}

Physical-chemical analysis (PCA) of condensed phase chemical systems has three major purposes [1]. PCA establishes the presence of an interaction between different components of a mixture, resolves the stoichiometry of the interaction, and determines thermodynamic characteristics of the resulting chemical processes, most importantly, equilibrium constants. Currently, PCA is among the most efficient means of determination of the stoichiometry of compounds formed by chemi- 
cal interaction in condensed matter [2,3]. In those cases when a formed compound cannot be isolated, PCA is the only method capable of producing compound's composition. To a large extent, this fact establishes a place for PCA among other physical chemical approaches [4-6].

A comparison between the experimental and theoretically calculated composition-property diagrams is a standard tool in PCA of many-component systems [1]. Various properties of such systems can be deduced from the experimental data and deviation of the experimental data from the theoretical prediction. A high sensitivity of the electro-optical Kerr effect (EOKE) to many types of inter-molecular interactions (IMI) is a key to its successful application in the investigation of many-component systems [7]. However, the complexity and ambiguity in the interpretation of the experimental data set up limits on the types of systems amenable to the EOKE analysis [8-13]. Noticeable progress has been made in the development and improvement of EOKE measurement techniques, mainly by minimizing the effect of the electric field on a system. Techniques using a pulsed electric field [12-17] and an alternating field [18] have emerged. Still, the theoretical difficulties in relating the measured Kerr constant to molecular concentrations and characteristics of individual molecules are not overcome. Drawbacks in equations describing internal fields in complex systems as well as limitations of the addition scheme in the description of systems with strong IMI are the primary theoretical obstacles.

A new approach to the studies of conducting systems is offered by the optical Kerr effect (OKE) that is measured by a direct application of the electric field to the sample, avoiding the electrodes [19]. While OKE is sensitive to the anisotropy of the optical polarizability tensor of molecules, EOKE is sensitive to both permanent and induced electric dipole moments [20]. Combined with measurements of other characteristics including dielectric permittivity, light scattering and molecular refraction, OKE permits determination of molecular properties, geometries and local structures of investigated media [21]. An excellent review of the application of OKE to pure liquids, liquid crystals, and plastic crystals can be found in Ref. [22]. References [23, 24] describe the principles and details of the experimental procedure and setup.

OKE is analogous to the electric Kerr effect that is observed in static and slowly varying fields. The differences between the two effects are essential, though. The frequency of the optical electric field is so high that the field does not change orientations of permanent molecular dipoles. Only induced dipole moments are important in OKE, in particular, since they determine the anisotropic part of the Kerr constant. Due to the high frequency of the applied electric field, OKE is extremely suitable for studies of aqueous solutions and other conducting systems. The magnitude of OKE is only linearly dependent on temperature. Therefore, OKE can be used to separate the linear term and to study temperature non-linearities of EOKE. 


\section{Application of Kerr constant $(B)$ in physical-chemical analysis of binary systems}

The measured Kerr constant of a pure substance can be expressed by the following general formula [25]:

$$
B_{\lambda}=\frac{1}{\lambda n} F(n, \varepsilon) \frac{N}{N_{A}} m K,
$$

where the function $F(n, \varepsilon)$ describing the intra-molecular field is given by one of the following expressions:

$$
\begin{aligned}
& F(n, \varepsilon)=\frac{\left[\left(n^{2}+2\right)(\varepsilon+2)\right]^{2}}{81} \quad \text { (Lorentz field), } \\
& F(n, \varepsilon)=\frac{\left(n^{2}+2\right)^{2}(\varepsilon+2)}{27} \quad(\text { Zamkov field), } \\
& F(n, \varepsilon)=\frac{\left(n^{2}+2\right)(\varepsilon+2)}{9} \quad \text { (Vuks field). }
\end{aligned}
$$

The measured Kerr constant of a binary mixture of non-interacting compounds is determined through addition scheme as

$$
\begin{aligned}
B_{\lambda_{1,2}} & =\frac{1}{\lambda n_{1,2}} F\left(n_{1,2} \varepsilon_{1,2}\right) \frac{N_{1,2}}{N_{A}}\left(x_{1} m K_{1}+x_{2} m K_{2}\right) \\
& =\frac{N_{1,2}}{n_{1,2}}\left[\frac{B \lambda_{1} n_{1}}{N_{1}} x_{1} \frac{F\left(n_{1,2}, \varepsilon_{1,2}\right)}{F\left(n_{1}, \varepsilon_{1}\right)}+\frac{B \lambda_{2} n_{2}}{N_{2}} x_{2} \frac{F\left(n_{1,2}, \varepsilon_{1,2}\right)}{F\left(n_{1}, \varepsilon_{1}\right)}\right] .
\end{aligned}
$$

The physical meaning of $F(n, \varepsilon)$ remains the same as for pure compounds. An expression for $F(n, \varepsilon)$ that is applicable to binary mixtures has been proposed in Ref. [26]

$$
F\left(n_{1,2}, \varepsilon_{1,2}\right)=\left(\frac{n^{2}+2}{3} \frac{\varepsilon+2}{3}\right)^{2 S} .
$$

With particular choices of the power $2 S$, Eq. (6) reduces to the above expressions for the Lorentz, Zamkov, and Vuks fields. In the Vuks-Zamkov model and according to the gas-phase theory of non-interacting binary mixtures the value of $2 S$ equals 1 . This value is close to 1.2 determined experimentally for the $\mathrm{CS}_{2}-\mathrm{CCl}_{4}$ binary mixture [12]. The physical meaning of the fractional power remains unclear.

An optimal form of the $F(n, \varepsilon)$ function applicable to binary systems with weak IMI has been developed in a paper [27] and is based on the internal field model of Scholte [28].

The approach developed in Ref. [10] considers the deviation of the Kerr constant of a binary mixture $\Delta B_{1,2}$ from the additive value calculated with a binary interaction potential. In this approach care must be taken with the definition of $\Delta B_{1,2}$ and the choice of $F(n, \varepsilon)$. In particular, within the limits of the gas phase theory of non-interacting binary systems, the molar Kerr constant of the binary 
mixture $m K_{1,2}$ is linearly dependent on the concentration of either of the components. At the same time, the concentration dependence of $B_{1,2}$ is not linear. The non-linearity must be taken into account in the calculations of the deviations of the measured $B_{1,2}$ from the "ideal" curve.

20 binary mixtures characterized by weak IMI have been studied in chloroform [12]. Based on the analysis of binary isotherms of Kerr constants, possible functional dependence of $B_{1,2}$ on the concentration of the second component $X_{2}$ has been considered. Figures 1-3 compare the experimentally determined property-composition data with those calculated by Eqs. $(5,6)$. The Kerr constants $B_{1,2}$ are plotted as functions of the molar fraction $X_{2}$ of the second component of the mixture. The power $2 S$ is chosen phenomenologically in order to minimize the deviation between the experimental and theoretical data. The theoretical procedure is described in the original work of Hellwart et al. [26]. Based on the presented data it is possible to conclude that theory and experiment agree well only for a limited number of weakly interacting systems. The power $2 S$ in the expression for the internal field (6) is close to 1 for binary systems dominated by

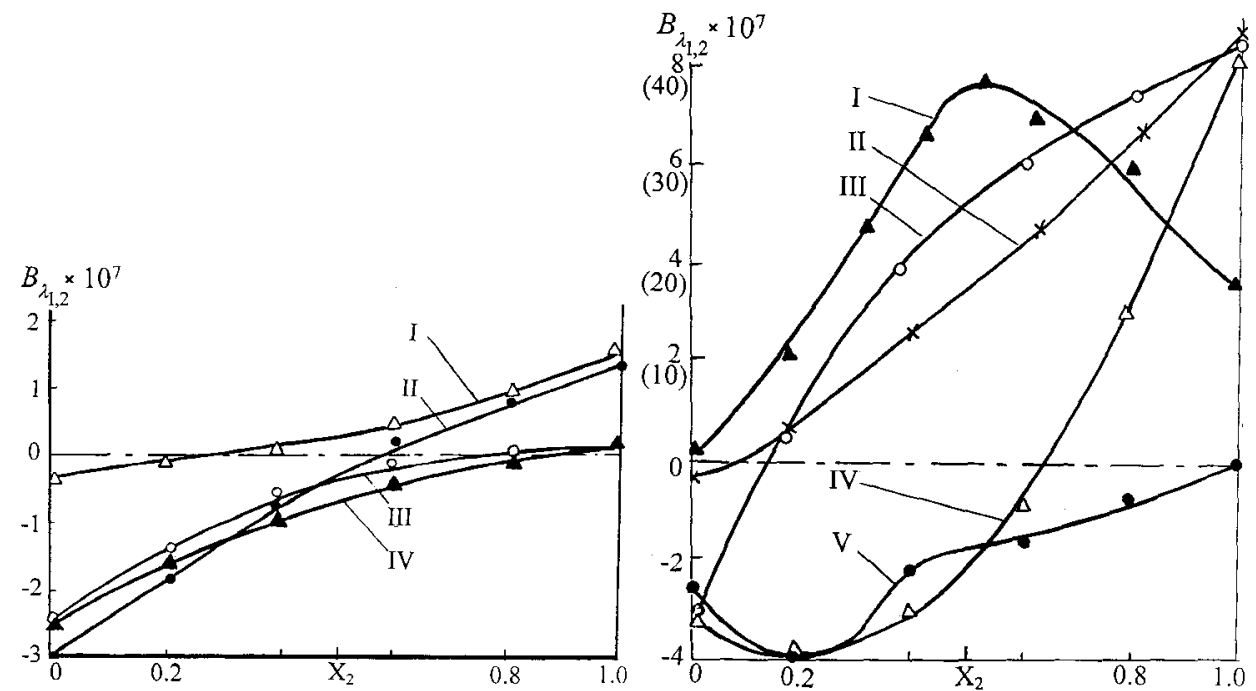

Fig. 1

Fig. 2

Fig. 1. $B_{1,2}$ dependence on the molar fraction of the second component of the following binary systems: I - chloroform-acetone $(2 S=0.3)$; II - chloroform-anizole $(2 S=2)$; III - chloroform-dioxane $(2 S=2)$; IV - chloroform-cyclohexane $(2 S=1.2)$.

Fig. 2. $\quad B_{1,2}$ dependence on the molar fraction of the second component of the following binary systems: I - chloroform-pyridine; II - chloroform-acetonitrile $(2 S=0.3)$;

III - chloroform-nitromethane $(2 S=0.6)$; IV - chloroform-dimethylaniline; $\mathrm{V}$ - chloroform-triethylamine. The $B_{1,2}$ scale for I and II is given in parentheses. 


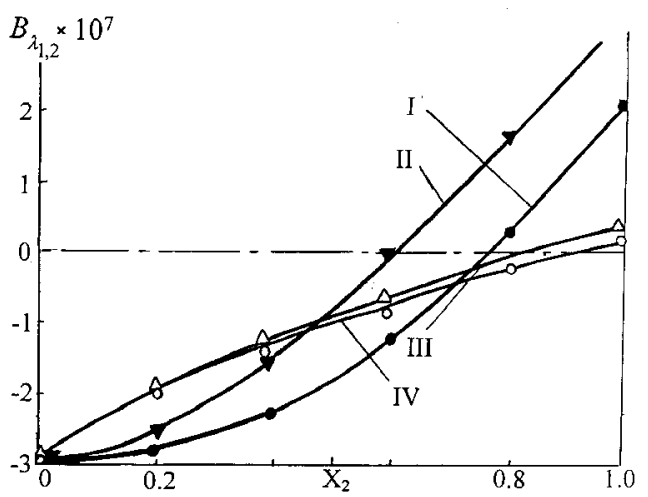

Fig. 3. $B_{1,2}$ dependence on the molar fraction of the second component of the following binary systems: I - chloroform-methylformiate $(2 S=2)$; II - chloroform-chloropropane $(2 S=2)$; III - chloroform-benzene $(2 S=1.2)$; $\mathrm{IV}-$ chloroform $-\mathrm{CCl}_{4}(2 S=1.2)$.

dispersive IMI. In the presence of a significant dipole-dipole contribution to IMI satisfactory results can be obtained using the above equations for the internal field with $2 S=2$. All other types of IMI, in particular those characteristics of liquids, lead to a sharp disagreement between the well-known expressions of the classical orientation theory and the experimental data. For instance, the experimental data for the mixtures containing acetonitrile, acetone, and nitromethane are best fitted with the $2 S$ values equal $0.3,0.3$, and 0.6 , respectively. Equation (6) fails to describe systems that form hydrogen bonds. This is the case for the mixtures of chloroform with pyridine, dimethylamine, and triethylamine. In general, complex formation can be taken into account by modification of the particle number density used in the calculation of the internal fields. However, this modification extends beyond the addition scheme. Thus, addition schemes using analytic forms of the internal field function $F(n, \varepsilon)$ generally fail to describe the experimental data even for systems with weak IMI. The internal field function can be modified empirically based on the deviations in the composition-property diagram between the theoretical values calculated by the addition scheme and the experimentally determined molar Kerr constants [29].

\section{Application of molar Kerr constant ( $m K$ ) in physical-chemical analysis of binary system}

The molar Kerr constant is determined from the following equation [12]:

$$
m K(x)=\frac{B_{\lambda}}{A N(\varepsilon+2) / 3},
$$

where $A=\frac{n^{2}+2}{3} \frac{1}{N_{A} \lambda n}$ is weakly dependent on $n$. Within a $5 \%$ error $n=\hat{n}$, and 
therefore $A \approx 0.025$ and $\hat{n}=1.35 \div 1.55$. The theoretical values of $m K_{1,2}$ in a series of chloroform, acetone, and water binary mixtures are calculated with the help of the equation

$$
B_{\lambda_{1,2}}=\frac{N_{1,2}}{N_{A} \lambda n_{1,2}}\left(\frac{n_{1,2}^{2}+2}{3} \frac{\varepsilon_{1,2}+2}{3}\right) m K_{1,2} .
$$

It is assumed that the molar Kerr constant of a binary mixture is not additive, i.e., $m K_{1,2} \neq x_{1} \cdot m K_{1}+x_{2} \cdot m K_{2}$, while $N_{1,2}=x_{1} N_{1}+x_{2} N_{2}$ and $\varepsilon_{1,2}=x_{1} \varepsilon_{1}+x_{2} \varepsilon_{2}$ are additive. Figures 4 and 5 show the experimental values of $B_{\lambda_{1,2}}$ together with the corresponding values of $m K_{1,2}$ : the $\Delta$ and $\bigcirc$ symbols denote the experimental data, while the lines are obtained using Eqs. $(5,6)$ and $(8)$. The dashed line gives the additive $m K_{1,2}$ values.

In the chloroform series the value of $2 S$ is close to 1 , and the dispersive IMI dominate. The deviations of the molar Kerr constants of the mixtures from the additive values are insignificant. It may be expected that the additivity in the molar Kerr constants of binary mixtures will be perturbed by an increasing contribution of the dipole-dipole interaction. Thus, in the cases where the experimental data can be described by Eq. (6) within the addition scheme, the $2 S$ values

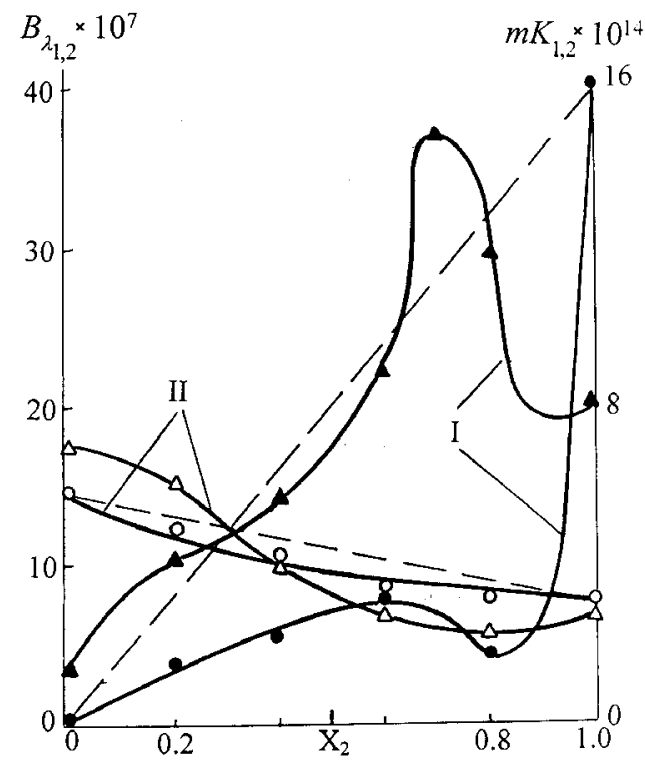

Fig. 4. $\quad B_{1,2}$ and $m K_{1,2}$ dependence on the molar fraction of the second component of the following binary systems: I - water-pyridine; II - acetone-acetic acid. $\Delta$ and $O$ denote the experimental values of $B_{1,2}$ and $m K_{1,2}$, respectively. The solid lines represent $B_{1,2}$ calculated according to $(5,6)$ and $m K_{1,2}$ calculated according to (8). The broken lines give the additive values of $m K_{1,2}$. 

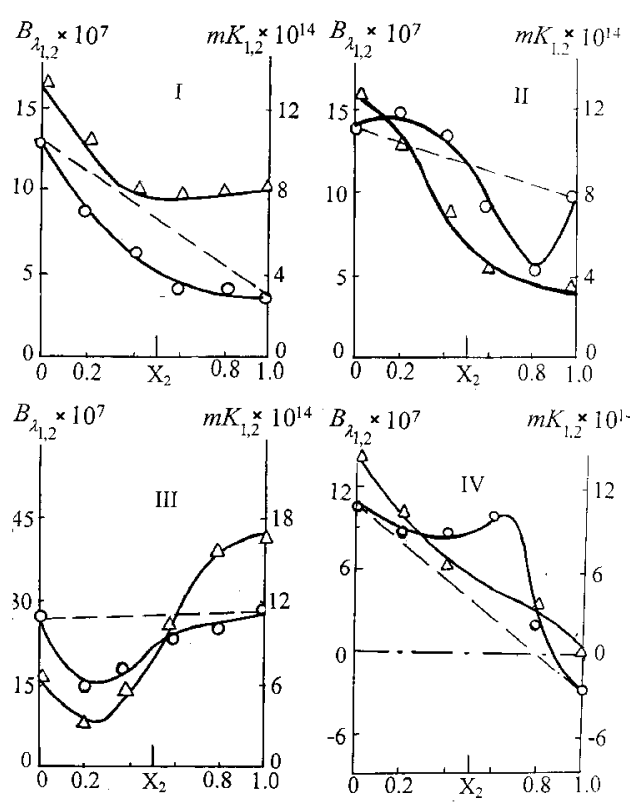

Fig. 5. $\quad B_{1,2}$ and $m K_{1,2}$ dependence on the molar fraction of the second component of the following binary systems: acetone-nitromethane (I); acetone-acetonitrile (II); acetone-chloropropane (III); acetone-triethylamine (IV). $\Delta$ and $\bigcirc$ denote the experimental values of $B_{1,2}$ and $m K_{1,2}$, respectively. The solid lines represent $B_{1,2}$ calculated according to $(5,6)$ and $m K_{1,2}$ calculated according to $(8)$. The broken lines give the additive values of $m K_{1,2}$.

characterize IMI. The $2 S$ value also provides a measure of additivity. According to [11], $2 S=1$ implies that the properties of a binary mixture are simple sums of the corresponding properties of the components. An absence of additivity suggests relatively strong IMI.

The following general description of the electro-optical properties of weakly interacting binary systems can be proposed. Consider a molar Kerr constant $m K_{1,2}$ of a binary mixture as a function of the molar fraction $X_{2}$ of the second component chosen so as $m K_{1}<m K_{2}$. The observed deviations of the experimental curve from the additive straight line can be positive, negative or a combination of both. Different types of deviations can be explained in the following manner. Suppose that $m K_{1,2}=x_{1} \cdot m K_{1}^{*}+x_{2} \cdot m K_{2}^{*}$, where $m K_{1}^{*}$ and $m K_{2}^{*}$ are functions of $X_{2}$ such that $m K_{1}^{*}\left(x_{2}=0\right)=m K_{1}$ and $m K_{2}^{*}\left(x_{2}=1\right)=m K_{2}$. The deviation is defined by $\Delta m K_{1,2}=x_{1} \cdot m K_{1}+x_{2} \cdot m K_{2}-m K_{1,2}=x_{1} \cdot m K_{1}+x_{2} \cdot m K_{2}-\left(x_{1} \cdot m K_{1}^{*}+x_{2} \cdot m K_{2}^{*}\right)$. Consider two limiting cases.

Case 1. Let $m K_{1}^{*}=m K_{1}=$ const and $m K_{2}^{*}=m K_{2} \cdot f\left(x_{2}\right)$. This corresponds to a complete absence of complex formation between molecules of components 
1 and 2. Polarizability of the first component is independent of the presence of the second component.

Suppose that $\mathrm{d} m K_{2}^{*}=\xi_{1} \cdot m K_{1}^{*} \mathrm{~d} x_{2}=\xi_{1} \cdot m K_{1} \mathrm{~d} x_{2}$, where $\xi_{1}$ is a parameter to be determined experimentally. Solution to this equation with the condition $m K_{2}^{*}\left(x_{2}=1\right)=m K_{2}$ has the form $m K_{2}^{*}=m K_{2}-\xi_{1} \cdot m K_{1}\left(1-x_{2}\right)$. Substitution of this expression into the equation for $\Delta m K_{1,2}$ gives $\Delta m K_{1,2}=\xi_{1} \cdot m K_{1} \cdot x_{2}\left(1-x_{2}\right)$. If $m K_{2}^{*}$ decreases with increasing $x_{1}$ (or decreasing $x_{2}$ ), then $\xi_{1} \cdot m K_{1}>0$ and $\Delta m K_{1,2}>0$. The deviation $\Delta m K_{1,2}$ is maximal at $x_{2}=0.5$ and reaches the value $\Delta m K_{1,2}^{\max }=\xi_{1} \cdot m K_{1} / 4$. Given the experimental value of $\Delta m K_{1,2}^{\max }$ it is trivial to estimate $\xi_{1}$. A general shape of the $m K_{1,2}\left(x_{2}\right)$ curve is given in Fig. 6a. Typically, the experimental curve is asymmetric due to a minor dependence of $m K_{1}^{*}$ on $x_{2}$. Examples of the experimental curves of this type can be found in the chloroform-methyl formiate data, where $\xi_{1} \cdot m K_{1} \approx 400$, as well as in the water and acetone series data. In the latter case it is the acetone $m K_{2}$ that is composition dependent. In the water series $m K_{2}$ of the second component, e.g., pyridin or acetic acid depends on $x_{2}$.
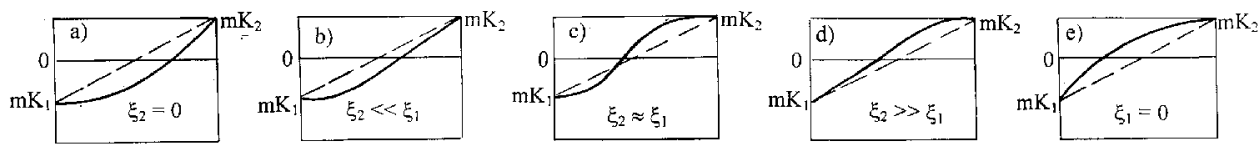

Fig. 6. Possible functional dependence of the molar Kerr constant $m K_{1,2}$ on composition $X_{2}$ of a binary mixture.

Case 2. Let $m K_{2}^{*}=m K_{2}=$ const and $m K_{1}^{*}=m K_{1} \cdot f\left(x_{2}\right)$. By the analysis similar to that of case 1 we have $\mathrm{d} m K_{1}^{*}=\xi_{2} \cdot m K_{2}^{*} \cdot \mathrm{d} x_{2}=\xi_{2} \cdot m K_{2} \cdot \mathrm{d} x_{2}$ with $\xi_{2} \cdot m K_{2}>0$ when $m K_{1}^{*}$ increases with increasing $x_{2}$. Solution to this equation under the condition $m K_{1}^{*}\left(x_{2}=0\right)=m K_{1}$ gives $m K_{1}^{*}=m K_{1}+\xi_{2} \cdot m K_{2} \cdot x_{2}$. The deviation $\Delta m K_{1,2}$ now becomes $\Delta m K_{1,2}=-\xi_{2} \cdot m K_{2} \cdot x_{2}\left(1-x_{2}\right)$. In this case $\Delta m K_{1,2}<0$. The shape of the curve $m K_{1,2}\left(x_{2}\right)$ is shown in Fig. 6b. With $x_{2}=0.5 \Delta m K_{1,2}=\Delta m K_{1,2}^{\max }-\xi_{2} \cdot m K_{2} / 4$, and the parameter $\xi_{2}$ can be easily determined. Similar to the case 1 , the experimental curve is asymmetric, since $m K_{2}^{*}$ slightly depends on $x_{2}$, and the deviation is maximal away from $x_{2}=0.5$. The curves of the second type are observed much more often than the curves of the first type. In particular, most of the chloroform mixtures fall into the second category. The composition dependence is present in the molar Kerr constant of chloroform, which is more sensitive to the dipolar environment.

General case, case 3 . This case includes the possibility of complex formation. $m K_{1}^{*}=m K_{1} \cdot f\left(x_{2}\right) ; m K_{2}^{*}=m K_{2} \cdot f\left(x_{2}\right)$. Under the assumption that $\mathrm{d} m K_{1}^{*}=\xi_{2} \cdot m K_{2} \cdot \mathrm{d} x_{2}, \mathrm{~d} m K_{2}^{*}=\xi_{1} \cdot m K_{1}^{*} \cdot \mathrm{d} x_{2}$, equations of the $\mathrm{d}^{2} y / \mathrm{d} x^{2}=c^{2} y$ type are obtained for $m K_{1}$ and $m K_{2}$, where $c^{2}=\xi_{1} \xi_{2}, x_{2}=x$ and $y$ is either $m K_{1}^{*}$ or $m K_{2}^{*}$. The sign in front of $c^{2}$ depends on the signs of $m K_{1}$ and $m K_{2}$. Solutions to these equations are hyperbolic if the sign in front of $c^{2}$ is 
positive: $y=c_{1} \sinh (c x)+c_{2} \cosh (c x)$, and periodic if the sign is negative: $y=$ $D_{1} \sin (c x)+D_{2} \cos (c x)$. With a proper choice of the boundary conditions relatively complicated expressions for constants $c_{1}, D_{1}, c_{2}, D_{2}$ can be obtained. These expressions are not transparent and are difficult to relate to experiment. The curves corresponding to the general case are illustrated in Figs. 6c-e. The curve shown in Fig. $6 \mathrm{~d}$ is rare. Determination of the $\xi_{1}, \xi_{2}$ parameters individually in the $\xi_{2} \gg \xi_{1}$ or $\xi_{1} \gg \xi_{2}$ limits is not particularly useful for the qualitative purposes. From the practical viewpoint it is sufficient to classify the experimental curves to one of the 3 cases described above.

According to the statistical theory of multi-component systems due to Kielich [30] the deviations of the experimentally determined molar Kerr constants of binary mixtures from the corresponding additive values can be numerically expressed by the following expansion:

$$
m K=\sum_{i} x_{i} \cdot m K_{i}+\sum_{i j} x_{i} \cdot x_{j} \cdot m K_{i j}+\sum_{i j k} x_{i} \cdot x_{j} \cdot x_{k} \cdot m K_{i j k}+\cdots,
$$

where the molar Kerr constants of all orders are composition independent. The first order constants $m K_{i}$ carry information about individual molecules. The second order constants $m K_{i j}$ describe the interaction of a molecule of the $i$-th compound with a molecule of compound $j$. The constants $m K_{i j k}$ describe the third order interactions of $i$ with $j$ and $k$, and so on. The second order constants $m K_{i j}$ depend on binary correlation functions $g_{i j}^{(2)}\left(\tau_{i}, \tau_{j}\right) ; m K_{i j k}$ depend on triple correlations $g_{i j k}^{(3)}\left(\tau_{i}, \tau_{j}, \tau_{k}\right)$, where $\tau_{i}, \tau_{j}, \tau_{k}$ represent translation and rotation coordinates of molecules $i, j, k$.

These molecular correlations are not needed for the description of the deviations from additivity in the presence of weak IMI. If stronger IMI take place, the correlation independent description becomes invalid and binary, triple, etc. correlation parameters reflecting the form and degree of IMI have to be introduced. It is reasonable to expect that the types of the correlation factors will not change for other magneto- and electro-optic effects. For instance, comparing Kerr effect measurements with those of the Cotton-Muton effect one can determine the first order deviations from additivity. Then, relationships of the experimentally determined $\xi_{1}$ and $\xi_{2}$ can be used to study stronger, nonlinear interactions between molecules in a mixture. A more rigorous justification of this statement requires additional experimental investigation.

The general form of the $B_{\lambda}\left(x_{2}\right)$ and $m K\left(x_{2}\right)$ diagrams characterizes IMI in a given system. For instance, the presence of the two maxima in the $B_{\lambda}\left(x_{2}\right)$ curve of the pyridine-water system, Fig. 4 , can be interpreted within the limits of the cluster theory by the following association mechanism. Initially with increasing concentration, pyridine molecules do not disrupt the short-range structure of the water lattice and associate only with the free water molecules located between the structured water clusters. This type of association corresponds to the first maximum on the $B\left(x_{2}\right)$ curve. A sharp increase of concentration fluctuations in 
the system and a light scattering maximum support the initial mechanism. With additional increases in the molar fraction of pyridine the short-range water structure is disrupted. Pyridin molecules are able to bind to the previously clustered water molecules. The overall anisotropy of the system is disrupted, and $B\left(x_{2}\right)$ decays. The subsequent growth of $B\left(x_{2}\right)$ is no longer related to the concentration fluctuations, but rather is due to a rapid increase in the dipolar term in the Kerr constant. The increase is observed when polar associates start decomposing into ionic pairs under the influence of the polar medium. The second maximum on the $B\left(x_{2}\right)$ curve corresponds to a maximum in $\varepsilon\left(x_{2}\right)$ observed within the same concentration range.

Schematically, the association in the pyridine-water system can be expressed

as

$$
\begin{aligned}
& \mathrm{Py}+\mathrm{H}_{2} \mathrm{O}_{\text {free }} \Rightarrow \mathrm{Py} \cdot \mathrm{H}_{2} \mathrm{O}, \\
& 2 \mathrm{Py}+\mathrm{H}_{2} \mathrm{O}_{\text {struct }} \Rightarrow \mathrm{Py}_{2} \cdot \mathrm{H}_{2} \mathrm{O} \Leftrightarrow \mathrm{Py}+\mathrm{OH}^{-}+\mathrm{PyH}^{+} .
\end{aligned}
$$

This association mechanism agrees well with the results of Vuks [31] and Fialkov et al. [32].

\section{Physical-chemical analysis of binary systems in solution, the isomolar series method}

PCA of binary systems with a strong donor-acceptor interaction, for instance, in the case of H-bonding, can be carried out in a sufficiently inert, typically nonpolar solvent. The analysis in this case is done by the method of isomolar series [33]. The equations used in the isomolar series EOKE approach properly account for the changes in the concentrations of solution components due to complex formation [34]. PCA is performed in the polarization-composition coordinates. Formally, both polarization and molar Kerr constant can be denoted by a general additive characteristic symbol $P$. The following equation holds:

$$
P_{A D C}=\left(P_{S A D}-P_{S} x_{S}^{0}\right) /\left(\tilde{x}_{A}^{0}+\tilde{x}_{D}^{0}\right),
$$

where the indices $A, D, S, C$ refer to the acceptor, donor, solvent, and complex, respectively. $\tilde{x}_{i}^{0}$ are the prepared molar fractions of the solution components, satisfying $\sum_{i=1}^{k} \tilde{x}_{i}^{0}=1$. Reference [34] suggests several methods for determination of the partial property $P_{C}$, including a graphical extrapolation and two versions of an analytic extrapolation in the isomolar series region. Under the assumptions that component $A$ is in excess of component $D$ and the molar fraction of the latter approaches 0 , the methods systematically account for the changes of the number of moles (particles) in the system due to the complex formation. The graphical extrapolation determines the compositions of the complexes by the extrema of the traditional expression $\Delta P_{A D C}=P_{A D C}-P_{A} x_{A}^{0}-P_{D} x_{A}^{0}$. With the excess of one of the complex components, equations $P_{A C}=\left(P_{S A C}-P_{S}\right) / \tilde{x}_{A C}+P_{S}, \tilde{x}_{A C}=$ 
$\left[\tilde{x}_{A}^{0}+\tilde{x}_{D}^{0}\left(1-\nu_{A}\right)\right] / \nu_{D} Z$, and $Z=\tilde{x}_{S}^{0}+\tilde{x}_{A}^{0}\left(1-\nu_{A}\right) / \nu_{D}$ are used to determine the values of the "apparently" additive property $P_{A C}$ of a binary system. As before, the indices $A, D, C, S$ refer to the acceptor and donor components $A, D$, complex $C$, and solvent $S . x_{A}^{0}, x_{D}^{0}$ are the initial molar fractions of components $A$ and $D$ in a binary mixture. $\tilde{x}_{i}^{0}$ are the molar fractions of the components of the solution in a tertiary mixture. $\nu_{A}$ and $\nu_{D}$ are the stoichiometric coefficients of the complex of a known composition. The graphical extrapolation of the $P_{C}$ values as functions of the molar fraction $x_{D}^{0}$ is carried out in the limit of $x_{D}^{0} \rightarrow 0$ and using the expression $P_{C}=\left(P_{A C}-P_{A}\right) \nu_{D} / x_{D}^{0}+P_{A}$. Polarization, molar Kerr constant or another additive property of the complex ${ }_{\infty} P_{C}=\lim P_{C}$ is obtained in this manner.

The graphical extrapolation of a property $P$ of a complex has the same drawbacks as the extrapolation of $P$ of individual compounds. Generally, it is not as accurate as an analytic extrapolation. Two versions of analytic extrapolation were developed [34]. According to the first version $\infty_{\infty} P_{C}=\left[{ }_{\infty} P^{\prime}-P_{S}\left(\nu_{A}+\nu_{D}\right)\right] / x_{A i}+$ $P_{S}\left(\nu_{A}+\nu_{D}\right)$, where $-\infty P^{\prime}=\lim _{x_{D}^{0} \rightarrow 0} \frac{Z P_{S A C}-P_{S A}}{x_{D}^{0}} \nu_{D}+\left(\nu_{A}+\nu_{D}\right) P_{S A}$, and $\tilde{x}_{A i}^{0}$ is the molar fraction of component $A$ in the origin of the isomolar series defined by $D=0$. The molar Kerr constant $P \equiv m K_{C}$ is given by

${ }_{\infty} P^{\prime} \equiv_{\infty}\left(m K^{\prime}\right)=K_{S A}\left[\frac{\bar{M}}{M_{S A}}+\left(\delta-\beta-\frac{2 \varepsilon_{S A}}{\varepsilon_{S A}+2} \alpha-\frac{4 n_{S A}^{2}}{n_{S A}^{2}+2} \gamma\right) \nu_{D}\right]$,

where $K_{S A}=6 \lambda n_{S A} B_{S A} M_{S A} /\left(n_{S A}^{2}+2\right)^{2}\left(\varepsilon_{S A}+2\right)^{2} d_{S A} ; M_{S A}=M_{S} \tilde{x}_{S}^{0}+M_{A} \tilde{x}_{A i}^{0}$; $\bar{M}=M_{S A}\left(\nu_{A}+\nu_{D}\right)+\tilde{x}_{A i}^{0}\left(M_{D}-M_{A}\right)$. The coefficients $\alpha, \beta, \gamma, \delta$ enter the expressions $\varepsilon_{S A C}=\varepsilon_{S A}\left(1+\alpha x D^{0}\right) ; d_{S A C}=d_{S A}\left(1+\beta x_{D}^{0}\right) ; n_{S A C}=n_{S A}\left(1+\gamma x_{D}^{0}\right)$; $B_{S A C}=B_{S A}\left(1+\delta x_{D}^{0}\right)$, and reflect the dependences of the dielectric constant $\varepsilon$, density $d$, refractive index of light $n$, and Kerr constant $B$ of the solution on the initial molar fraction $x_{D}^{0}$. The latter approaches zero, i.e., $A \gg D$.

The second version of the analytic extrapolation is based on the assumption that with a significant excess of component $A$ in the $S+A+D$ system and a complete suppression of the $A_{m} D_{n}$ complex dissociation, the concentration dependence of dielectric permittivity, density, refraction index, and Kerr constants $B$ of a solution can be expressed by $\varepsilon_{S A K}=\varepsilon_{S}\left(1+\alpha_{A} \tilde{x}_{A}+\alpha_{C} \tilde{x}_{C}\right)$. As a result, $\alpha_{C}$ is determined by equation $\alpha_{C}=\left.\frac{\nu_{A}}{\varepsilon_{S}} \frac{\partial \varepsilon_{S A C}}{\partial \tilde{x}_{A}^{0}}\right|_{X_{B}^{0} \rightarrow 0}+\alpha_{A}\left[1+\left(\nu_{A}+\nu_{B}-1\right) \tilde{x}_{S}^{0}\right]$. Parameters $\beta_{C}, \gamma_{C}$, and $\delta_{C}$ are determined similarly. Parameters $\alpha_{A}, \beta_{A}, \gamma_{A}, \delta_{A}$, characteristic of solutions of individual components are obtained by the well-known approach [35].

The molar Kerr constant $\infty\left(m K_{C}\right)$ of a complex is calculated by the following formula due to Briegleb [36]:

$$
\infty\left(m K_{C}\right)=m K_{S}\left(\frac{M_{C}}{M_{S}}-\beta_{C}-\frac{2 \varepsilon_{S}}{\varepsilon_{S}+2} \alpha_{C}+\frac{4 n_{S}^{2}}{n_{S}^{2}+2} \gamma_{C}+\delta_{C}\right),
$$

where $m K_{S}=6 \lambda n_{S} B_{S} M_{S} /\left(n_{S}^{2}+2\right)^{2}\left(\varepsilon_{S}+2\right)^{2} d_{S} ; M_{C}=M_{A} \nu_{A}+M_{D} \nu_{D}$. 
A practical application of the extrapolation methods is illustrated with the pyridine $-p$-chlorophenol system in heptane [34]. The extrapolated results are compared with the data of other approaches. Satisfactory agreement in the ${ }_{\infty}\left(m K_{C}\right)$ values obtained by all methods is observed.

The isomolar series method produces reliable data when complexes are thermodynamically stable. If this is not the case, complex dissociation should be accounted for in the calculation of the ${ }_{t} P_{C}$ values. Typically, the dissociation increases ${ }_{t} P_{C}$. Reference [37] modifies the first version of analytic extrapolation to include the possibility of dissociation. Complexes of the 1:1 composition are treated in Ref. [37] explicitly. The dissociation of complexes of other compositions can be handled in a similar manner.

The ${ }_{t} P_{C}$ values of the 1:1 complexes are defined in terms of the ${ }_{\infty} P_{C}$ values by the following expression:

$$
{ }_{t} P_{C}=\left[{ }_{\infty} P_{C}-\left(1-\omega_{0}\right)\left(P_{A}+P_{D}\right)\right] / \omega_{0}
$$

where $P_{A}$ and $P_{D}$ are either molar polarizations or molar Kerr constants of the original components. $\omega_{0}=\lim _{D \rightarrow 0}[D] /\left[D_{0}\right]$ is the degree of complexing of component $D$ in the limit of $x_{D} \rightarrow 0$. The values of $\omega_{0}$ are calculated from $\omega_{0}=k \sqrt{(2-k) / k}$, where $k=\tilde{x}_{A 1}^{0}\left[{ }_{\infty} P_{C}-\left(P_{A}+P_{D}\right)\right] / 2 \Delta P, \tilde{x}_{A 1}^{0}$ is the concentration of component $A$ in the origin of the isomolar series. $\Delta P$ is the deviation

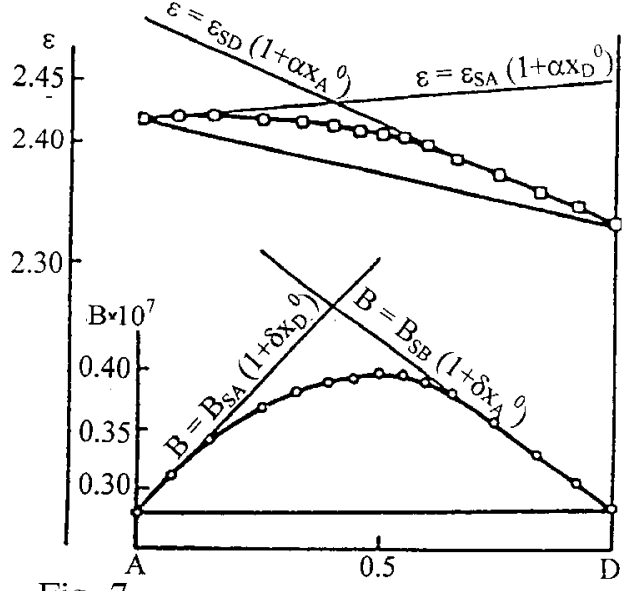

Fig. 7

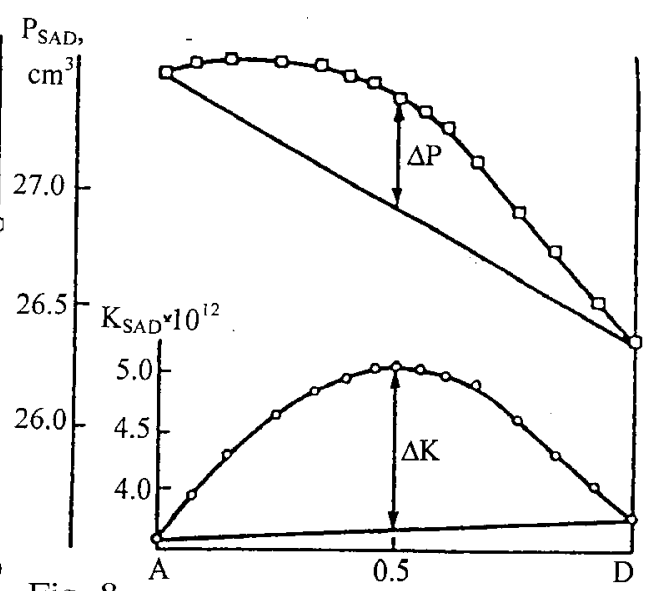

Fig. 8

Fig. 7. The isomolar series determination of the $\alpha, \delta$ parameters for the $N$-methylimidazole (A) $-p$-chlorophenol (D) system in dioxane.

Fig. 8. $P_{S A D}$ and $K_{S A D}$ as functions of composition of the $N$-methylimidazole (A) $p$-chlorophenol (D) system in dioxan. The plots illustrate determination of $\Delta P$ and $\Delta K$ in the calculation of $K_{C}$ of the complexes. 
of property $P$ from additivity. The deviation is calculated for a tertiary system at its maximum. The values of $\omega_{0}$ produce thermodynamic stability constants $K_{C}$ according to the equation $K_{C}=\omega_{0} /\left(1-\omega_{0}\right) \tilde{x}_{A 1}^{0} N$, where $N$ is the total molality of the initial solution in the isomolar series.

The isomolar series approach to the determination of ${ }_{t} P_{C}$ and $K_{C}$ is illustrated by the $N$-methylimidazole $-p$-chlorophenol system in dioxane, Figs. 7 and 8 . The predicted maximal degree of complex formation is only $60-63 \%$ for this example [37], but equals $87 \%$ in the pyridin - $p$-chlorophenol system in heptane. These results agree with the corresponding data obtained by independent physical-chemical approaches.

\section{Internal field of liquids}

An adequate description of the internal field in a condensed phase dielectric is a prerequisite that is often missing preventing a wider application of EOKE in PCA.

The available models of the internal field can be classified into two categories. The first one encompasses the approaches that use the Lorentz functional form of the internal field and vary the field contribution to the theoretical expression for the Kerr constant. Approaches of the second kind replace the Lorentz function by a function that follows from a more sophisticated model of the internal field [38]. Thus, the theory of Onsager [39] leads to the following expression for the molar Kerr constant:

$$
m K=\frac{1}{(1-a f)(1-b g)}\left(m K^{0}-\frac{2 \pi N}{9} a f \Theta_{1}\right),
$$

where $f=2(\varepsilon-1) / r^{3}(2 \varepsilon+1), g=2\left(n^{2}-1\right) / r^{3}\left(2 n^{2}+1\right), r$ is a characteristic molecular radius, $K^{0}$ is the gas phase molar Kerr constant, $a$ and $b$ are some constants, and $\Theta_{1}$ is the anisotropic term of the Kerr constant. The molar Kerr constant in the Onsager model can be calculated knowing the molar Kerr constant in the Lorentz model and the macroscopic parameters of the medium ( $\varepsilon$ and $n$ ) by the formula: $m K$ (Ons.) $=m K$ (Lor.) $\left[\frac{\left(2 \varepsilon+n^{2}\right)(\varepsilon+2)}{3 \varepsilon\left(n^{2}+2\right)}\right]^{2}$. More general theories account for molecular asymmetry. The theory of Scholte [28] gives the following expression for the molar Kerr constant [40]:

$$
\begin{aligned}
m K & =\frac{2 \pi N}{9}\left[\frac{1}{45 k T} \frac{1}{(1-a f)(1-b g)} \sum\left(b_{i i} m_{i i}-b_{j j} m_{j j}\right)\left(a_{i i} k_{i i}^{2}-a_{j j} k_{j j}^{2}\right)\right. \\
& \left.+\frac{1}{45 k^{2} T^{2}} \frac{1}{(1-a f)(1-b f)} \sum\left(b_{i i} m_{i i}-b_{j j} m_{j j}\right)\left(\mu_{i}^{2} k_{i i}^{2}-\mu_{j}^{2} k_{j j}^{2}\right)\right] .
\end{aligned}
$$

Here, $m_{i i}=\left(2 n^{2}+1\right) /\left[3 n^{2}+3\left(1-n^{2}\right) O i\right] ; k_{i i}=(2 \varepsilon+1) /\left[3 \varepsilon+3(1-\varepsilon) O_{i}\right] ; O_{i}$ is the ellipsoid constant $[41,42]$. Applications of the ellipsoid model to solutions can be found in Ref. [42]. 
Equations of this type contain parameters that are hard to determine. The theories that work within the limits of the Lorentz internal field defined in terms of the $\left(n^{2}+2\right) / 3$ and $(\varepsilon+2) / 3$ functions avoid the parameterization problem. The phenomenological theory of the Kerr effect created by Zamkov [43-45] considers the energy of a molecule subject to an electric field and leads to the following equation for the Kerr constant

$$
B=\frac{\pi N_{1}}{3}\left(\frac{n^{2}+2}{3}\right)^{2} \frac{\varepsilon+2}{3}\left(\Theta_{1}+\Theta_{2}\right),
$$

which is different from the ordinary expression only by the first power of the $(\varepsilon+2) / 3$ function. A more general expression [45] includes the internal field due to Bottcher

$$
\begin{aligned}
B= & \pi N_{1} \frac{n^{2}+2}{n^{2}}\left[\left(1-\frac{f}{1-f b} \frac{\mu^{2}}{3 k T}\right)^{2} \frac{9 \varepsilon^{2}}{(2 \varepsilon+1)^{2}(1-f b)^{2}} \Theta_{3}\right. \\
& \left.+\frac{\varepsilon(\varepsilon+2)}{3(2 \varepsilon+1)(1-f b)}\left(\Theta_{1}+\frac{\Theta_{2}}{1-f b}\right)\right],
\end{aligned}
$$

where $\Theta_{2}$ is the dipolar and $\Theta_{3}$ is the Voigt term. A similar result has been obtained by Vuks by consideration of the Lorentz-Lorenz equation for the condensed media $[31,46]$. The analysis of Vuks shows that a straightforward replacement of the external field in the gas phase by the internal field in the liquid phase is not acceptable and that the first power of the $(\varepsilon+2) / 3$ factor is required.

The differences in the internal field functions stipulate the changes in the extrapolation formulas used in the experimental determination of the molar Kerr constant [25]

$$
\infty(m K)_{2}=m K_{1}\left(\frac{M_{1}}{M_{2}}-\beta-\frac{2 \varepsilon_{1}}{\varepsilon_{1}+2} \alpha-\frac{2 n_{1}^{2}-4}{n_{1}^{2}+2} \gamma+\delta\right) .
$$

Differentiation of the Vuks-Zamkov equation [46] leads to the following expression:

$$
\infty(m K)_{2}=m K_{1}\left(\frac{M_{1}}{M_{2}}-\beta-\frac{\varepsilon_{1}}{\varepsilon_{1}+2} \alpha+\frac{4}{n^{2}+2} \gamma+\delta\right) .
$$

A systematic analysis of the variations of the internal field model obtained within the limits of various theories as well as an assessment of the Kerr constant data that follows from these theories can be found in Refs. [47-49].

The assumption that the polarizability ellipsoid, dipole moment, and hyperpolarizability parameters are molecular constants independent of density, pressure, temperature, and solvent forms the foundation of the physical-chemical methods based on molecular optics. It is clear that the assumption holds only approximately. IMI directly affect molecular electronic structure and, therefore, change molecular polarizability and other molecular properties. It is necessary to find ways of elimination of the many-body by either a calculation or additional measurements. Condensed phase theories of the Kerr constant that account for correlation of 
molecular orientation form a separate category. The statistical-mechanical theory of Buckingham [50-53] is one example. This theory explicitly considers IMI as well as the dependence of molecular polarity and polarizability on the field strength. Typically, theories of this type involve complicated models and produce parameters that are difficult to determine. As a result, application of these theories to the calculation of the polarizability anisotropy is rather complicated.

The hyperpolarizability contributions to the Kerr constant have been estimated in Refs. [54, 55]. An attempt to develop a more complete theory of the Kerr effect in condensed media that accounts for the hyperpolarizability contributions, orientation correlations, IMI, electrostriction and the Joule effect has been carried out by Proutiere [56]. The traditional spherical model has led to the equation

$$
B=\frac{2 \pi N_{1}}{\lambda n}\left(\frac{n^{2}+2}{3}\right)^{2} \frac{\varepsilon+2}{3}\left(10 \Gamma+\frac{20}{135 k T} \beta \mu+\Theta_{1}+\Theta_{2}\right)
$$

Here, $\beta$ and $\Gamma$ are the spherically averaged first and second hyperpolarizabilities. The temperature independent term can be neglected in the investigation of strongly anisotropic molecules, for example, aromatic compounds.

Reference [7] presents a comparative analysis of the main approaches to the description of the internal field in the calculation of the Kerr constant. The calculated molar Kerr constants obtained for the gas $\left(m K^{\mathrm{G}}\right)$ and liquid $\left(m K^{\mathrm{L}}\right)$ phases have been investigated using the following theories: the classical Langevene-Born theory with the Lorentz field $\left(m K_{\mathrm{L}}^{\mathrm{L}}\right)$, the theories of Vuks $\left(m K_{\mathrm{V}}^{\mathrm{L}}\right)$ and Zamkov $\left(m K_{\mathrm{Z}}^{\mathrm{L}}\right)$, the theories based on the Onsager $\left(m K_{\mathrm{O}}^{\mathrm{L}}\right)$ and Scholte fields $\left(m K_{\mathrm{S}}^{\mathrm{L}}\right)$ as well as the Kielich procedure involving a Taylor series expansion in the solute concentration $\left(m K_{\mathrm{K}}^{\mathrm{L}}\right)$. It is found that $m K_{\mathrm{S}}^{\mathrm{L}}, m K_{\mathrm{V}}^{\mathrm{L}}, m K_{\mathrm{Z}}^{\mathrm{L}}$ give best results. All models under study are ranked as follows: $m K_{\mathrm{L}}^{\mathrm{L}} \approx m K_{\mathrm{K}}^{\mathrm{L}}<m K_{\mathrm{O}}^{\mathrm{L}} \approx m K_{\mathrm{S}}^{\mathrm{L}}<$ $m K_{\mathrm{V}}^{\mathrm{L}} \approx m K_{\mathrm{Z}}^{\mathrm{L}}<m K^{\mathrm{G}}$.

In a series of papers [57-62] we have developed expressions for the Kerr constants of liquids and molar Kerr constants of solutes that are based on the classical Langeven-Born theory. The following additional factors have been accounted for: the anisotropy of the electric field of the probe light wave, the reactive field of Onsager molecular moments interacting with the medium, the differences between molecular dipoles in the liquid and gas phases. The expressions employ Onsager internal fields that have been modified for polar liquids. Applications of the derived equations for the Kerr constant to real systems and tests of the modified cavity and reactive field expressions indicate that the approach has a good potential for further development and application of the Kerr effect in PCA.

\section{Conclusions}

Application of the Kerr effect to the physical-chemical analysis of binary systems provides reliable information about intermolecular interactions. When interactions are strong, an extension of the additive approach and account for molecular 
correlations are required. The experimental data agree with the statistical theory of the Kerr effect due to Kielich. The theory concludes that the deviations of the molar Kerr constants of binary mixtures from the additive values are generally determined by molecular correlations, whose order depends on the specific character of intermolecular interaction.

Application of the electro-optical Kerr effect to the investigation of binary systems in inert solvents provides both structures of donor-acceptor complexes, and equilibrium constants for complex formation.

Further progress in the physical-chemical applications of the electro-optical Kerr effect depends on a proper description of the internal fields of liquids and improvements in the theories of optical properties of molecular aggregates.

\section{References}

[1] Yu.Ya. Fialkov, Physical-Chemical Analysis of Liquid Systems and Solutions, Naukova Dumka, Kiev 1992, p. 245 (in Russian).

[2] A.K. Yakhkind, M.N. Polanski, J. Opt. Technol. 67, 501 (2000).

[3] J.A. Boutin, P.H. Lambert, S. Bertin, J.P. Volland, J.L. Fauchere, J. Chromatogr. $B$ 725, 17 (1999).

[4] E.A. Guggenheim, Mixtures, Clarendom Press, Oxford 1952, p. 270.

[5] N.Y. Turova, E.P. Turevskaya, M.I. Yanovskaya, A.I. Yanovski, V.G. Kessler, D.E. Tcheboukov, Polyhedron 17, 899 (1998).

[6] A.Y. Ananov, Mol. Biology 24, 473 (1990).

[7] V.V. Prezhdo, M.V. Khashchina, V.A. Zamkov, Electro-Optical Investigations in Physics and Chemistry, Vyshcha Schkola, Kharkov 1982, p. 152 (in Russian).

[8] R. Konopka, S. Surma, Acta Phys. Pol. A 31, 1075 (1967).

[9] M.T. Ratzsch, F. Rickelt, H. Rosner, Z. Phys. Chem. Leipz. 255, 933 (1974).

[10] A.E. Lutski, V.D. Berestetskaya, V.V. Prezhdo, Zh. Obshch. Khim. 43, 2462 (1973).

[11] R. Hellwarth, N. George, Optoelectronics 1, 213 (1963).

[12] M.V. Khashchina, P.L. Pakhomov, V.V. Prezhdo, Dokl. AN USSR 250, 146 (1980).

[13] J.M. Neto, A.B. Villaverdte, J. Phys. Condens. Matter 8, 2791 (1996).

[14] J.M. Neto, E. Munin, A.B. Villaverde, Meas. Sci. Technol. 2, 1026 (1991).

[15] R.K. Khanna, P.K. Sharma, Czech. J. Phys. 38, 345 (1988).

[16] R.K. Khanna, E. Dempsey, G.P. Jones, J. Phys. E, Scientific 11, 1210 (1978).

[17] K. Kikuchi, G. Honda, H. Watanabe, J. Phys. E, Scientific 21, 568 (1988).

[18] V.A. Zamkov, Instr. Exp. Techn. 20, 1182 (1977).

[19] A.M. Paillette, J. Chimie Phys. 65, 1629 (1968).

[20] S. Kielich, Acta Phys. Pol. A 30, 683 (1966).

[21] Z. Blaszczak, Acta Phys. Pol. A 71, 601 (1987). 
[22] Z. Blaszczak, M. Farhoud, J. Mol. Liquids 68, 157 (1996).

[23] Z. Blaszczak, H. Malek, Acta Phys. Pol. A 69, 621 (1986).

[24] Z. Blaszczak, M. Farhoud, J. Mol. Liquids 62, 209 (1994).

[25] G. Briegleb, Z. Phys. Chem. B 14, 97 (1931).

[26] R. Helwarth, N. George, Opto-Electronics 1, 213 (1969).

[27] M.T. Ratzsch, R. Patz, Setzungsber. Akadem. Wiss. DDR, Math.-Naturwiss.-Techn. 4, 21 (1978).

[28] T. Scholte, Physica 15, 450 (1949).

[29] V.V. Prezhdo, I.N. Krainov, Intermolecular Interactions and Electric Properties of Molecules, Osnova, Kharkov 1994, p. 240 (in Russian).

[30] S. Kielich, Mol. Phys. 6, 49 (1963).

[31] M.F. Vuks, Optika i Spektrosk. 21, 697 (1966).

[32] Yu.Ya. Fialkov, Yu.Ya. Borovikov, Elektrokhim. 1, 1106 (1965).

[33] E.N. Gur'yanova, I.P. Goldschtein, I.P. Romm, Donor-Acceptor Bond, Khimiya, Moscow 1973, p. 382. (in Russian).

[34] S.B. Bulgarievich, V.S. Bolotnikov, O.A. Osipov, Zh. Obshch. Khim. 47, 139 (1977).

[35] C.G. Le Fevre, R.J.W. Le Fevre, J. Chem. Soc. , 4041 (1953).

[36] G. Briegleb, Z. Phys. Chem. B 16, 682 (1933).

[37] S.B. Bulgarewich, V.S. Bolotnikov, O.A. Osipov, Zh. Obshch. Khim. 48, 1338 (1978).

[38] C.V. Raman, K.S. Krishnan, Proc. R. Soc. Lond. A 117, 579 (1928).

[39] L. Onsager, J. Am. Chem. Soc. 58, 486 (1936).

[40] C.J.F. Bottcher, Theory of Electric Polarization, Elsevier, Amsterdam 1952, p. 492 .

[41] J.A. Osborn, Phys. Rev. 67, 351 (1945).

[42] H.G. Kuball, R. Gob, Z. Phys. Chem. Neue Folge 63, 251 (1969).

[43] V.A. Zamkov, Ukrain. Fiz. Zh. 7, 720 (1962).

[44] V.A. Zamkov, Optika i Spektrosk. 15, 654 (1963).

[45] V.A. Zamkov, in: Optical Investigation in Liquids and Solutions, Ed. Ya.Yu. Akhadov, Fan, Tashkent 1965, p. 128 (in Russian).

[46] M.F. Vuks, in: Optical Investigation in Liquids and Solutions, Ed. Ya.Yu. Akhadov, Fan, Tashkent 1965, p. 133 (in Russian).

[47] H.G. Kuball, R. Gob, Z. Phys. Chem. Neue Folge 62, 237 (1968).

[48] R. Gob, H.G. Kuball, Z. Phys. Chem. Neue Folge 72, 153 (1970).

[49] S. Kielich, Mol. Phys. 6, 49 (1963).

[50] A.D. Buckingham, R.E. Raab, J. Chem. Soc., 2341 (1957).

[51] A.D. Buckingham, Proc. Phys. Soc. A 68, 910 (1953).

[52] A.D. Buckingham, Trans. Faraday Soc. 52, 611 (1956).

[53] A.D. Buckingham, P.J. Stiles, G.L.D. Ritchie, Trans. Faraday Soc. 67, 577 (1971). 
[54] A.D. Buckingham, J.A. Pople, Proc. Phys. Soc. A 68, 905 (1953).

[55] A.D. McLean, M. Yoshimine, J. Chem Phys. 47, 1927 (1967).

[56] A. Proutiere, Contribution a l'etude de la conformationale de compose aromatique, Univ. Bordeaux, Bordeaux 1973, p. 164.

[57] V.V. Prezhdo, G.V. Tarasova, O.V. Prezhdo, S.A. Tyurin, O.N. Akulova, T.N. Kurskaya, Acta Phys. Pol. A 84, 253 (1993).

[58] V.V. Prezhdo, G.V. Tarasova, O.V. Prezhdo, S.A. Tyurin, O.N. Akulova, T.N. Kurskaya, Acta Phys. Pol. A 85, 509 (1994).

[59] V.V. Prezhdo, G.V. Tarasova, O.V. Prezhdo, S.A. Tyurin, O.N. Akulova, T.N. Kurskaya, Acta Phys. Pol. A 88, 419 (1995).

[60] V.V. Prezhdo, G.V. Tarasova, O.V. Prezhdo, S.A. Tyurin, O.N. Akulova, T.N. Kurskaya, Acta Phys. Pol. A 85, 797 (1994).

[61] V.V. Prezhdo, G.V. Tarasova, O.V. Prezhdo, S.A. Tyurin, O.N. Akulova, T.N. Kurskaya, Acta Phys. Pol. A 86, 327 (1994).

[62] V.V. Prezhdo, G.V. Tarasova, O.V. Prezhdo, S.A. Tyurin, O.N. Akulova, N.I. Ivanov, Acta Phys. Pol. A 89, 47 (1996). 